\title{
Optimal Joint Allocation of MultiSlot Spectrum Sensing and Transfer Power in MultiChannel Cognitive Radio
}

\author{
Xin Liu, ${ }^{1,2}$ Min Jia, ${ }^{2}$ Xuemai Gu, ${ }^{2}$ Fanqiang Kong, ${ }^{1}$ and Qingfeng Jing ${ }^{1}$ \\ ${ }^{1}$ College of Astronautics, Nanjing University of Aeronautics and Astronautics, Nanjing 210016, China \\ ${ }^{2}$ Communication Research Centre, Harbin Institute of Technology, Harbin 150080, China \\ Correspondence should be addressed to Xin Liu; liuxinstar1984@nuaa.edu.cn and Min Jia; jiamin@hit.edu.cn
}

Received 15 April 2014; Revised 14 July 2014; Accepted 23 July 2014; Published 21 August 2014

Academic Editor: Fanli Meng

Copyright (c) 2014 Xin Liu et al. This is an open access article distributed under the Creative Commons Attribution License, which permits unrestricted use, distribution, and reproduction in any medium, provided the original work is properly cited.

\begin{abstract}
In multichannel, cognitive radio (CR), the secondary user (SU) is allowed to utilize multiple subaltern frequency bands of the primary user (PU), when these bands, namely, subchannels are not currently being used. To support this spectrum reuse functionality, the SU is required to sense each subchannel, and only the subchannels wherein the PU is inactive are available for the spectrum access of the SU. In this paper, a multislot spectrum sensing and transfer scheme for multichannel CR is proposed, whose sensing stage is divided into several time slots allocated to the subchannels for spectrum sensing. While guaranteeing the spectrum sensing performance on each subchannel and limiting the interference to the PU, we formulate an optimization problem that maximizes the SU's aggregate throughput by jointly allocating the optimal number of sensing time slots and the optimal transfer power to each subchannel. Theoretical analysis is given to prove the feasibility of the proposed optimization problem and simulation results are presented to show the notable improvement on the SU's throughput when the sensing time slots and the transfer power are both optimized by the proposed scheme.
\end{abstract}

\section{Introduction}

Cognitive radio (CR) has been proposed to overcome the shortage of spectrum resources that is introduced by the traditional fixed spectrum allocation method [1]. In CR, the secondary user (SU) can transfer data through an idle channel that is licensed to the primary user (PU) but currently unoccupied, providing that the PU's normal communication is not disturbed $[2,3]$. Hence, the spectrum sensing to detect the PU's activity is an essential method to reuse the unoccupied spectrum and has become one of the key technologies in CR [4].

Spectrum sensing requests the SU to efficiently and accurately detect the presence of the PU, in order to avoid causing harmful interference to the PU [5]. Since, to the SU, the PU's signal is often with unknown location, structure, and strength, energy detection, which is widely used due to its simple implementation, serves as the optimal spectrum sensing method when the detector only receives the power of the received signal [6]. The sensing time has deep impact on the performance of energy detection, and the sensing performance, reflected by the false alarm and detection probabilities, can be improved with the increase of the sensing time [7]. In CR, the higher the detection probability is, the less interference the PU suffers, while the lower the false alarm probability becomes, the more opportunity the SU gets to access the spectrum [8].

In recent years, multichannel CR has been proposed to improve the throughput by allowing the SU to utilize multiple idle subchannels to transfer data synchronously; however, before communicating, the SU has to detect the PU's activity in each subchannel [9]. A multiband joint detection method is introduced in [10], which jointly detects the PU's signals over multiple frequency bands rather than over one band in the equal sensing time. In [11], the authors consider an orthogonal frequency division multiplexing (OFDM) based multichannel CR system and present efficient algorithms to maximize the SU's aggregate throughput by jointly optimizing both the sensing threshold and the power allocation, taking into account the influence of the false alarm and detection 


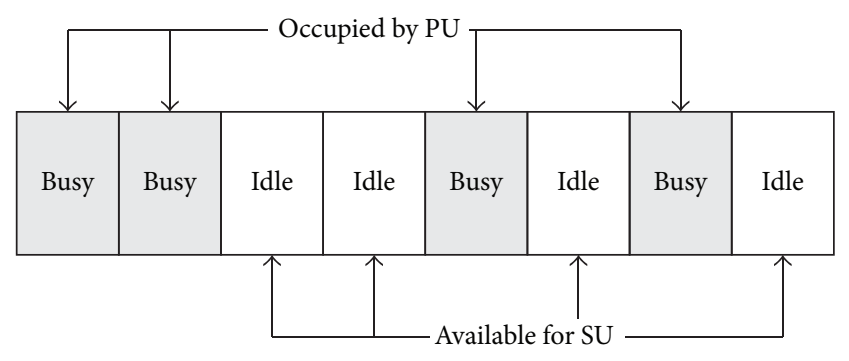

FIgURE 1: Multichannel CR model.

probabilities. However, the sensing time is not considered by the authors, which may shorten the SU's transfer time and decrease its throughput. In $[12,13]$, a sensing-throughput tradeoff problem is analyzed mathematically, and the energy detection is used to prove that the formulated problem indeed has one optimal sensing time that yields the highest throughput. However, in multichannel CR, how to obtain the optimal set of the sensing time to all the subchannels is very important.

In this paper, subject to the constraints of the false alarm and detection probabilities of each subchannel and the aggregate interference power and rate of the SU, a multislot spectrum sensing and transfer scheme for multichannel CR is formulated. The SU's aggregate throughput is maximized by jointly allocating the optimal number of sensing time slots and the optimal transfer power to each subchannel. The analytical and numerical results obtained in this paper clearly show that the maximal throughput can be achieved when the sensing time slots and the transfer power are both optimized.

\section{Preparation}

2.1. Multichannel Cognitive Radio. In multichannel CR, the SU may operate over multiple subchannels synchronously; therefore the SU's throughput can be improved greatly. Consider a PU system, based on multicarrier modulation, operating over a wideband spectrum that is divided into $L$ nonoverlapping narrowband subchannels. Since the PU may not use all the subchannels to transfer in the specific time and area, the left unused subchannels are available for the SU's spectrum access, as shown in Figure 1. Before accessing some subchannel, the SU must estimate the PU's state (presence or absence) in the subchannel by performing spectrum sensing, and only when the PU's absence is detected, the SU can use this subchannel to transfer data [14]. However, if the PU's presence is undetected, the SU may also use the subchannel by causing harmful interference to the PU.

2.2. SU's Spectrum Sensing. In spectrum sensing, since the $\mathrm{SU}$ and the PU are two different kinds of communication systems, SU is difficult to obtain any communication information from the PU system. Hence, the energy detection is widely used in the SU's spectrum sensing, which can be directly implemented without obtaining any signal information. Energy detection firstly calculates the energy of the received signal and then compares it to a preset threshold [15].
If the PU is absent, only the noise is received by the SU's detector, while if the PU is present, the mixture of the noise and the PU's signal is received. Hence, according to the different activities of the PU, the SU's received signal $y_{l}$ in subchannel $l$ for $l=1,2, \ldots, L$ is given as follows:

$$
\begin{array}{r}
y_{l}(m)= \begin{cases}n_{l}(m), & H_{0}, \\
h_{l} x_{l}(m)+n_{l}(m), & H_{1},\end{cases} \\
m=1,2, \ldots, M_{l},
\end{array}
$$

where the states $H_{0}$ and $H_{1}$ denote the PU's absence and presence, respectively, $x_{l}(m)$ is the PU's signal with the power of $p_{l}^{x}, n_{l}(m)$ is the Gaussian white noise with the power of $\sigma_{l}^{2}, h_{l}$ is the subchannel gain between the PU and the SU, and $M_{l}$ is the number of sampling nodes. By supposing that the sensing time and the sampling frequency are $\tau_{l}$ and $f_{s}$, respectively, $M_{l}$ is given by

$$
M_{l}=\tau_{l} f_{s} .
$$

By averaging the energy of $M_{l}$ sampling nodes, the energy $\Omega_{y_{l}}$ of the received signal $y_{l}$ is obtained as follows:

$$
\Omega_{y_{l}}=\frac{1}{M_{l}} \sum_{m=1}^{M_{l}}\left\|y_{l}(m)\right\|^{2} .
$$

Obviously, $y_{l}(1), y_{l}(2), \ldots, y_{l}(M)$ are independently and identically distributed, and according to the Central Limit Theorem, with larger $M_{l}, \Omega_{y_{l}}$ obeys the Gaussian distribution approximately. Under the states $H_{i}$ for $i=0,1$, the mean $E_{y_{l}, H_{i}}$ and the variance $\sigma_{y_{l}, H_{i}}^{2}$ of $\Omega_{y_{l}}$ are given as follows:

$$
\begin{array}{cl}
E_{y_{l}, H_{0}}=\sigma_{l}^{2}, & \sigma_{y_{l}, H_{0}}^{2}=\frac{1}{M_{l}} \sigma_{l}^{4}, \\
E_{y_{l}, H_{1}}=\left(1+\gamma_{l}\right) \sigma_{l}^{2}, & \sigma_{y_{l}, H_{1}}^{2}=\frac{1}{M_{l}}\left(1+\gamma_{l}\right)^{2} \sigma_{l}^{4},
\end{array}
$$

where the sensing SNR $\gamma_{l}=h_{l}^{2} p_{l}^{x} / \sigma_{l}^{2}$. Energy detection compares $\Omega_{y_{l}}$ to the detection threshold $\lambda$. And the PU's presence is decided with $\Omega_{y_{l}} \geq \lambda$, while the subchannel is determined to be idle and available with $\Omega_{y_{l}}<\lambda$. If the PU is really absent but the PU's presence is falsely detected, the false alarm is produced, while if the PU is really present but the PU's absence is falsely detected, the miss detection happens. According to (2) and (4), the false alarm probability $P_{l}^{f}$ and the detection probability $P_{l}^{d}$ are given as follows:

$$
\begin{gathered}
P_{l}^{f}=Q\left(\frac{\lambda-E_{y_{l}, H_{0}}}{\sigma_{y_{l}, H_{0}}}\right)=Q\left(\left(\frac{\lambda}{\sigma_{l}^{2}}-1\right) \sqrt{\tau_{l} f_{s}}\right), \\
P_{l}^{d}=Q\left(\frac{\lambda-E_{y_{l}, H_{1}}}{\sigma_{y_{l}, H_{1}}}\right)=Q\left(\left(\frac{\lambda}{\sigma_{l}^{2}\left(1+\gamma_{l}\right)}-1\right) \sqrt{\tau_{l} f_{s}}\right),
\end{gathered}
$$

where $Q(x)=(1 / \sqrt{2 \pi}) \int_{x}^{\infty} e^{-\left(t^{2} / 2\right)} \mathrm{d} t$. According to (5), with a given $P_{l}^{d}, P_{l}^{f}$ can be denoted by $P_{l}^{d}$ as follows:

$$
P_{l}^{f}=Q\left(Q^{-1}\left(P_{l}^{d}\right)\left(1+\gamma_{l}\right)+\gamma_{l} \sqrt{\tau_{l} f_{s}}\right) .
$$




\begin{tabular}{|c|c|c|c|c|c|}
$\longleftarrow$ & \multicolumn{7}{c}{ Sensing $\longrightarrow$ Transfer $\longrightarrow$} \\
\hline 1 & 2 & 3 & $\ldots$ & $l$ & $l$ \\
\hline
\end{tabular}

FIGURE 2: Frame structure of single-channel CR.

\begin{tabular}{|c|c|}
$\longleftarrow$ Sensing_Transfer- & 1 \\
\hline 1 & 2 \\
\hline 2 & 3 \\
\hline 3 & $\cdots$ \\
\hline$\ldots$ & $L$ \\
\hline
\end{tabular}

FIGURE 3: Frame structure of single-slot multichannel CR.

\section{System Model}

3.1. Related Work. In CR, the SU's frame structure is often divided into sensing and transfer stages. In the sensing stage, the SU detects each subchannel and judges which one can be used, while, in the transfer stage, the SU uses the detected idle subchannels to transfer data.

In the sensing stage of [12], the SU searches an idle subchannel from the $L$ PU's subchannels one by one, and once an idle subchannel $l$ is found, the SU will stop sensing and transfer data in subchannel $l$ through the transfer stage, as shown in Figure 2. This scheme is named as single-channel CR.

In the sensing stage of [16], the bandwidth is divided into $L$ subbands, and each subband is allocated to sense one subchannel. Hence, the SU can use all the sensing time and part of the frequency band to sense a subchannel, as shown in Figure 3. In the transfer stage, the SU may use all the detected idle subchannels to transfer data. This scheme is named as single-slot multichannel CR.

In the sensing stage of [10], each subchannel is allocated to the equal continuous time slot to sense spectrum, and in one time slot, only one subchannel is detected by the SU, as shown in Figure 4. In the transfer stage, all the detected idle subchannels are available for the spectrum access of the SU. This scheme is named as equal-slot multichannel CR. However, the gain of each subchannel is often different, and the sensing performance can be further improved if we allocate more sensing time to the subchannel with better sensing gain.

3.2. Proposed Scheme. In this paper, the sensing stage is further divided into many small time slots, as shown in Figure 5. In each time slot, the SU can sense any subchannel with energy detection, and the number inside each time slot means the subchannel index to be sensed. Note that in the example, the SU senses spectrum firstly in channel 2, then in channels 1 and 3, and so forth, and finally in channels $L-1$ and $L$. Actually, the sensing order of the subchannels does not affect the final sensing performance of each subchannel. At the end of the sensing stage, the SU can obtain the used statuses of the $L$ subchannels by combining the sensing information in each time slot. The number of the sensing time

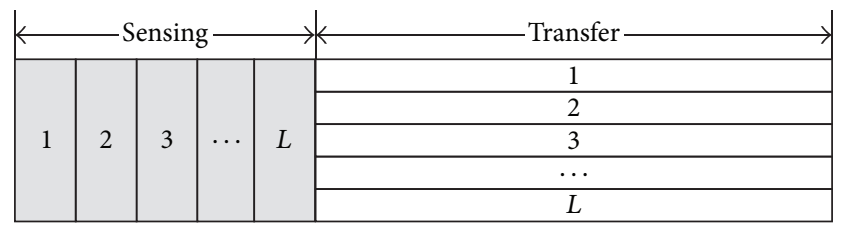

FIGURE 4: Frame structure of equal-slot multichannel CR.

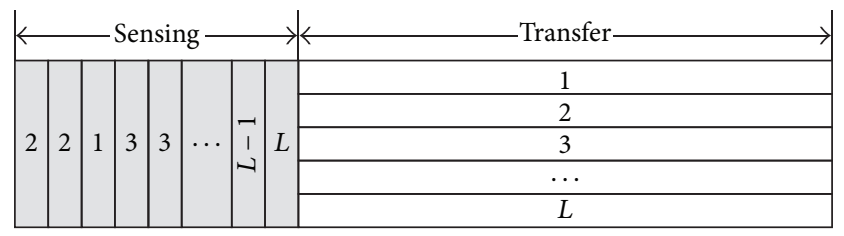

FIGURE 5: Proposed frame structure of multichannel cognitive radio.

slots allocated to each subchannel can be different, which is chosen according to the subchannel gain.

By supposing that the frame period is $T$, the length of each time slot is $\xi$, and the number of the time slots to sense subchannel $l$ is $k_{l}$, the sensing time of subchannel $l$ is given by

$$
\tau_{l}=k_{l} \xi
$$

and the SU's transfer time is given by

$$
t_{w}=T-\xi \sum_{l=1}^{L} k_{l}
$$

By assuming that the subchannels are symmetrical, the SU's data transfer in subchannel $l$ includes the following two cases.

(a) If the PU's absence is detected accurately by the SU in the probability of $1-P_{l}^{f}$, the SU can transfer in the subchannel effectively, with the rate of

$$
r_{l}^{0}=\log _{2}\left(1+\frac{p_{l} g_{l}^{2}}{\sigma_{l}^{2}}\right)
$$

where $g_{l}$ is the subchannel gain between the SU's transmitter and receiver.

(b) If the PU's presence is undetected in the miss detection probability of $1-P_{l}^{d}$, the SU may use the same subchannel with the PU and cause severe interference to the PU, with the rate of

$$
r_{l}^{1}=\log _{2}\left(1+\frac{p_{l} g_{l}^{2}}{\sigma_{l}^{2}\left(1+\gamma_{l}\right)}\right)
$$

Obviously, $r_{l}^{0}>r_{l}^{1}$. In case (b), if the $\mathrm{PU}$ is really present but the SU detects the PU's absence falsely in the probability of $1-P_{l}^{d}$, the SU will transfer data with the power of $p_{l}$ that causes severe interference to the PU. Hence, the SU's aggregate interference power is given by

$$
p_{I}=\sum_{l=1}^{L}\left(1-P_{l}^{d}\right) p_{l} h_{l}^{2}
$$


$p_{I}$ should be constrained by letting $p_{I} \leq I_{\max }$, where $I_{\max }$ is the maximal interference power suffered by the PU. Moreover in case (b), the SU transfers data with the rate of $r_{l}^{1}$ at the presence of the PU; these data may also disturb the PU's normal communication, and therefore we can get the maximal aggregate interference rate of the SU as follows:

$$
r_{I}=\sum_{l=1}^{L}\left(1-P_{l}^{d}\right) r_{l}^{1}=\sum_{l=1}^{L}\left(1-P_{l}^{d}\right) \log _{2}\left(1+\frac{p_{l} g_{l}^{2}}{\sigma_{l}^{2}\left(1+\gamma_{l}\right)}\right) .
$$

It is also necessary to constrain the SU's interference rate as $r_{I} \leq r_{I}^{\max }$. Since $1-P_{l}^{d} \leq 1$, we have

$$
r_{I} \leq \sum_{l=1}^{L} \log _{2}\left(1+\frac{\left(1-P_{l}^{d}\right) p_{l} g_{l}^{2}}{\sigma_{l}^{2}\left(1+\gamma_{l}\right)}\right)
$$

As $\log _{2}(x)$ is a convex function, we get the Cauchy inequality as follows:

$$
\begin{array}{r}
\frac{\log \left(x_{1}\right)+\log \left(x_{2}\right)+\cdots+\log \left(x_{n}\right)}{n} \\
\leq \log \left(\frac{x_{1}+x_{2}+\cdots+x_{n}}{n}\right) .
\end{array}
$$

By supposing $\gamma_{\min }=\min \left\{\gamma_{1}, \gamma_{2}, \ldots, \gamma_{L}\right\}$, from (13) and (14), we may derive the inequality as follows:

$$
\begin{aligned}
& \frac{1}{L} \sum_{l=1}^{L} \log _{2}\left(1+\frac{\left(1-P_{l}^{d}\right) p_{l} \zeta_{l}^{2}}{1+\gamma_{l}}\right) \\
& \quad \leq \log _{2}\left(1+\frac{1}{L} \sum_{l=1}^{L} \frac{\left(1-P_{l}^{d}\right) p_{l} \zeta_{l}^{2}}{1+\gamma_{l}}\right) \\
& \quad \leq \log _{2}\left(1+\frac{\sum_{l=1}^{L}\left(1-P_{l}^{d}\right) p_{l} \zeta_{l}^{2}}{L\left(1+\gamma_{\min }\right)}\right),
\end{aligned}
$$

where $\zeta_{l}=g_{l} / \sigma_{l}$. Hence, we can guarantee $r_{I} \leq r_{I}^{\max }$ by letting

$$
L \log _{2}\left(1+\frac{\sum_{l=1}^{L}\left(1-P_{l}^{\mathrm{d}}\right) p_{l} \zeta_{l}^{2}}{L\left(1+\gamma_{\min }\right)}\right) \leq r_{I}^{\max }
$$

from which we further obtain that

$$
\sum_{l=1}^{L}\left(1-P_{l}^{d}\right) p_{l} \zeta_{l}^{2} \leq \theta_{\max }
$$

where $\theta_{\max }=L\left(2^{r_{I}^{\max } / L}-1\right)\left(1+\gamma_{\min }\right)$.

In each frame, the SU may transfer data in cases (a) and (b) with the rates of $r_{l}^{0}$ and $r_{l}^{1}$ at the absence and presence of the PU, respectively, during the transfer time $t_{w}$. The SU's aggregate throughput over $L$ subchannels is given as follows:

$$
C=t_{w} \sum_{l=1}^{L}\left(r_{l}^{0}\left(1-P_{l}^{f}\right) P_{H_{0}}+r_{l}^{1}\left(1-P_{l}^{d}\right) P_{H_{1}}\right)
$$

where $P_{H_{0}}$ and $P_{H_{1}}$ are the appearance probabilities of the states $H_{0}$ and $H_{1}$, respectively.

In this paper, our goal of jointly allocating sensing time slots and transfer power is to maximize the SU's aggregate throughput subject to the constraints on the false alarm probability, the detection probability and the transfer power of each subchannel, and the aggregate interference power and the aggregate interference rate of the SU, as follows:

$$
\begin{aligned}
& \max _{\mathbf{k}, \mathbf{p}} C(\mathbf{k}, \mathbf{p}) \\
& P_{l}^{f} \leq \alpha \leq 0.5, \\
& \text { s.t. } \quad P_{l}^{d} \geq \beta \geq 0.5 \text {, } \\
& p_{l} \geq 0 \text {, } \\
& l=1,2, \ldots, L \text {; } \\
& \sum_{l=1}^{L} k_{l} \leq\left\lceil\frac{T}{\xi}\right\rceil ; \quad \sum_{l=1}^{L} p_{l} \leq p_{\max } \\
& \sum_{l=1}^{L}\left(1-P_{l}^{d}\right) p_{l} h_{l}^{2} \leq I_{\max } ; \quad \sum_{l=1}^{L}\left(1-P_{l}^{d}\right) p_{l} \zeta_{l}^{2} \leq \theta_{\max }
\end{aligned}
$$

where the vectors $\mathbf{k}=\left\{k_{1}, k_{2}, \ldots, k_{L}\right\}$ and $\mathbf{p}=\left\{p_{1}\right.$, $\left.p_{2}, \ldots, p_{L}\right\}, \alpha$ is the upper bound of the false alarm probability, $\beta$ is the lower bound of the detection probability, $p_{\max }$ is the SU's maximal transfer power, and $\lceil\phi\rceil$ denotes the maximal integer no bigger than $\phi$. In (19), we set $\alpha \leq 0.5$ and $\beta \geq 0.5$, because the SU's sensing performance must be good enough in order to decrease the interference to the PU and improve the spectrum access of the SU, as indicated in CR.

3.3. Solution to Optimization Problem. Equation (19) is a multiparameter optimization problem about $\mathbf{k}$ and $\mathbf{p}$, which can be solved by the alternating direction optimization (ADO) [16]. In each iteration, one of the two parameters is fixed while the other one is optimized, and the two parameters are alternately optimized through some iterations until both of them tend to be constant.

Firstly, we fix $\mathbf{p}$ (corresponding $r_{l}^{0}$ and $r_{l}^{1}$ are both fixed) and optimize $\mathbf{k}$. Since $P_{l}^{f}$ monotonically increases with the improvement of $P_{l}^{d}$, by substituting $P_{l}^{d} \geq \beta$ into (6), the lower bound of $P_{l}^{f}$ is given by

$$
P_{l}^{f} \geq Q\left(\eta_{l}^{0}+\eta_{l}^{1} \sqrt{k_{l}}\right)
$$

where $\eta_{l}^{0}=Q^{-1}(\beta)\left(1+\gamma_{l}\right)$ and $\eta_{l}^{1}=\gamma_{l} \sqrt{\xi f_{s}}$.

From (18), since $C$ improves with the increase of both $P_{l}^{f}$ and $P_{l}^{d}$, it can achieve the maximum only when $P_{l}^{d}=\beta$, and correspondingly, the equation of (20) is obtained. By substituting (20) into $P_{l}^{f} \leq \alpha$, we get $Q\left(\eta_{l}^{0}+\eta_{l}^{1} \sqrt{k_{l}}\right) \leq \alpha$, from which we obtain $k_{l} \geq q_{l}$, where $q_{l}$ is given by

$$
q_{l}= \begin{cases}0, & \alpha \geq Q\left(\eta_{l}^{0}\right), \\ \left\lfloor\left(\frac{Q^{-1}(\alpha)-\eta_{l}^{0}}{\eta_{l}^{1}}\right)^{2}\right\rfloor, & \alpha<Q\left(\eta_{l}^{0}\right),\end{cases}
$$


We suppose that the aggregate number of sensing time slots $K=\sum_{l=1}^{L} k_{l}$, where $K \geq \sum_{l=1}^{L} q_{l}$ and $K \in Z$. With the fixed $\mathbf{p}$, the constraints of (19) about $\mathbf{p}$ can be ignored, while, with $k_{l} \geq q_{l}$, the constraints of false alarm and detection probabilities can be satisfied. Hence, (19) transforms the suboptimization problem about $\mathbf{k}$ as follows:

$$
\begin{aligned}
\max _{\mathbf{k}} \quad C(\mathbf{k})=(T-\xi K) & \\
& \times\left(r_{l}^{0} P_{H_{0}}\left(1-Q\left(\eta_{l}^{0}+\eta_{l}^{1} \sqrt{k_{l}}\right)\right)\right. \\
& \left.\quad+r_{l}^{1} P_{H_{1}}(1-\beta)\right)
\end{aligned}
$$

In the following proposition, we will prove that $C(\mathbf{k})$ is a convex function about the vector $\mathbf{k}$, which denotes that there is an optimal set of $\mathbf{k}$ that maximizes $C(\mathbf{k})$.

Proposition 1. The objective function $C(\mathbf{k})$ is convex in $\mathbf{k}$.

Proof. Taking the second-order derivative of $C(\mathbf{k})$ in $k_{l}$ for $l=1,2, \ldots, L$ as follows:

$$
\begin{aligned}
\frac{\partial^{2} C(\mathbf{k})}{\partial^{2} k_{l}}= & -\frac{r_{l}^{0} P_{H_{0}} \eta_{l}^{1}}{4 \sqrt{2 \pi k_{l}}} \exp \left(-\frac{\left(\eta_{l}^{0}+\eta_{l}^{1} \sqrt{k_{l}}\right)^{2}}{2}\right) \\
& \times\left(\xi+\frac{\eta_{l}^{1}(T-\xi K)}{\sqrt{k_{l}}}\left(\eta_{l}^{0}+\eta_{l}^{1} \sqrt{k_{l}}+\frac{1}{\eta_{l}^{1} \sqrt{k_{l}}}\right)\right), \\
l & =1,2, \ldots, L,
\end{aligned}
$$

as $P_{l}^{f} \leq \alpha \leq 0.5$, by substituting (20), we have $Q\left(\eta_{l}^{0}+\eta_{l}^{1} \sqrt{k_{l}}\right) \leq$ 0.5 , from which $\eta_{l}^{0}+\eta_{l}^{1} \sqrt{k_{l}} \geq 0$ is obtained. Hence, according to (23), we have $\partial^{2} C(\mathbf{k}) / \partial^{2} k_{l} \leq 0$ for $l=1,2, \ldots, L$, which denotes that $C(\mathbf{k})$ is convex in $\mathbf{k}$.

If $K$ is given, since $\mathbf{k}$ is an integer vector, the optimal $k_{l}$ for $l=1,2, \ldots, L$ can be obtained by the greedy algorithm, as shown in Figure 4. In this figure, $G_{l}\left(k_{l}\right)$ is denoted by

$$
G_{l}\left(k_{l}\right)=r_{l}^{0} P_{H_{0}}\left(1-Q\left(\eta_{l}^{0}+\eta_{l}^{1} \sqrt{k_{l}}\right)\right)+r_{l}^{1} P_{H_{1}}(1-\beta) .
$$

As $K$ is an integer within a specific range, it is not complicated to search through $K$. With any given integer $K \in$ $\left[\sum_{l=1}^{L} q_{l},\lceil T / \xi]\right]$, we calculate $C(\mathbf{k})$ with the optimal vector $\mathbf{k}$ obtained by the greedy algorithm, and then we choose the optimal $K$ that maximizes $C(\mathbf{k})$, as follows:

$$
K^{*}=\underset{\sum_{l=1}^{L} q_{l} \leq K \leq\lceil T / \xi\rceil}{\arg \max } C(\mathbf{k}) .
$$

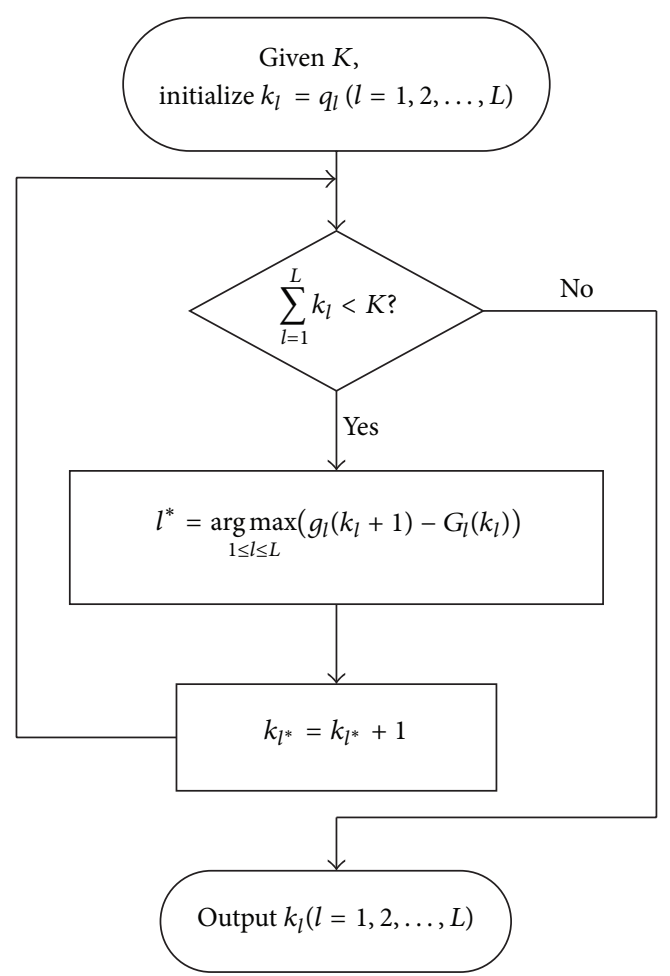

FIGURE 6: Flowchart to solve $k_{l}$ based on greedy algorithm.

With the given $K$, since the increment range of $\sum_{l=1}^{L} k_{l}$ is from $\sum_{l=1}^{L} q_{l}$ to $K$, the time complexity of the greedy algorithm in Figure 6 is $O\left(\left(K-\sum_{l=1}^{L} q_{l}\right) \log _{2} L+L\right)$. From (25), by enumerating $K \in\left[\sum_{l=1}^{L} q_{l},\lceil T / \xi\rceil\right]$, the aggregate time complexity to search an optimal set of $\mathbf{k}$ is given by

$$
F_{\mathrm{GE}}=O\left(\sum_{K=\sum_{l=1}^{L} q_{l}}^{\lceil T / \xi\rceil}\left(\left(K-\sum_{l=1}^{L} q_{l}\right) \log _{2} L+L\right)\right) .
$$

Secondly, we fix $\mathbf{k}$ (corresponding $t_{w}, P_{l}^{f}$, and $P_{l}^{d}$ are all fixed) and optimize $\mathbf{p}$. With the fixed $\mathbf{k}$, the constraints of false alarm and detection probabilities can be ignored. Noting $P_{l}^{d}=\beta,(19)$ transforms the suboptimization problem about $\mathbf{p}$ as follows:

$$
\begin{gathered}
\max _{p} \quad C(\mathbf{p})=\sum_{l=1}^{L}\left(\psi_{l}^{0} \log _{2}\left(1+\frac{p_{l} g_{l}^{2}}{\sigma_{l}^{2}}\right)\right. \\
\left.\quad+\psi_{l}^{1} \log _{2}\left(1+\frac{p_{l} g_{l}^{2}}{\sigma_{l}^{2}\left(1+\gamma_{l}\right)}\right)\right) \\
\text { s.t. } \quad \sum_{l=1}^{L} p_{l} \leq p_{\max } \\
\quad \sum_{l=1}^{L} p_{l} h_{l}^{2} \leq \frac{I_{\max }}{1-\beta} ; \quad \sum_{l=1}^{L} p_{l} \zeta_{l}^{2} \leq \frac{\theta_{\max }}{1-\beta} \\
p_{l} \geq 0, \quad l=1,2, \ldots, L,
\end{gathered}
$$


where $\psi_{l}^{0}=t_{w} r_{l}^{0} P_{H_{0}}$ and $\psi_{l}^{1}=t_{w} r_{l}^{1} P_{H_{1}}$. Using the Lagrange multiplier algorithm to solve (27), we obtain the Lagrange function as follows:

$$
\begin{aligned}
& L\left(\mathbf{p}, \lambda_{1}, \lambda_{2}, \lambda_{3}\right) \\
& =-\sum_{l=1}^{L}\left(\psi_{l}^{0} \log _{2}\left(1+\frac{p_{l} g_{l}^{2}}{\sigma_{l}^{2}}\right)+\psi_{l}^{1} \log _{2}\left(1+\frac{p_{l} g_{l}^{2}}{\sigma_{l}^{2}\left(1+\gamma_{l}\right)}\right)\right) \\
& \quad+\lambda_{1}\left(\sum_{l=1}^{L} p_{l}-p_{\max }\right)+\lambda_{2}\left(\sum_{l=1}^{L} p_{l} h_{l}^{2}-\frac{I_{\max }}{1-\beta}\right) \\
& \quad+\lambda_{3}\left(\sum_{l=1}^{L} p_{l} \zeta_{l}^{2}-\frac{\theta_{\max }}{1-\beta}\right)
\end{aligned}
$$

where $\lambda_{1}, \lambda_{2}$, and $\lambda_{3}$ are the Lagrange multipliers. According to the Karush-Kuhn-Tucker (KKT) conditions, the optimal p is got by letting $\partial L / \partial \mathbf{p}=0$, and if $\lambda_{1}, \lambda_{2}$, and $\lambda_{3}$ are not zero, they are got by letting the corresponding constraints acquire equations. Hence, we obtain

$$
\begin{gathered}
\lambda_{1}\left(\sum_{l=1}^{L} p_{l}-p_{\max }\right)=0, \\
\lambda_{2}\left(\sum_{l=1}^{L} p_{l} h_{l}^{2}-\frac{I_{\max }}{1-\beta}\right)=0, \\
\lambda_{3}\left(\sum_{l=1}^{L} p_{l} \zeta_{l}^{2}-\frac{\theta_{\max }}{1-\beta}\right)=0, \\
\frac{\partial L}{\partial p_{l}}=0 \text { for } l=1,2, \ldots, L .
\end{gathered}
$$$$
p_{l} \geq 0 \quad \text { for } l=1,2, \ldots, L, \quad \lambda_{1} \geq 0, \quad \lambda_{2} \geq 0, \quad \lambda_{3} \geq 0,
$$

Noting $p_{l} \geq 0$, direct calculation $\partial L / \partial p_{l}=0$ yields

$$
\begin{gathered}
p_{l}\left(\lambda_{1}, \lambda_{2}, \lambda_{3}\right) \\
=\left[\sqrt{\left(\frac{\psi_{l}^{0}+\psi_{l}^{1}}{2 \omega_{l}\left(\lambda_{1}, \lambda_{2}, \lambda_{3}\right)}\right)^{2}+\frac{\gamma_{l} \sigma_{l}^{2}\left(\psi_{l}^{0}-\psi_{l}^{1}\right)}{2 g_{l}^{2} \omega_{l}\left(\lambda_{1}, \lambda_{2}, \lambda_{3}\right)}+\frac{\gamma_{l}^{2} \sigma_{l}^{4}}{g_{l}^{4}}}\right. \\
\left.+\frac{\psi_{l}^{0}+\psi_{l}^{1}}{2 \omega_{l}\left(\lambda_{1}, \lambda_{2}, \lambda_{3}\right)}-\frac{\left(2+\gamma_{l}\right) \sigma_{l}^{2}}{2 g_{l}^{2}}\right]^{+} \\
l=1,2, \ldots, L
\end{gathered}
$$

where $[\cdot]^{+}=\max (\cdot, 0)$ and $\omega_{l}\left(\lambda_{1}, \lambda_{2}, \lambda_{3}\right)=\lambda_{1}+\lambda_{2} h_{l}^{2}+\lambda_{3} \zeta_{l}^{2}$. Using the subgradient method, we can further obtain $\lambda_{1}, \lambda_{2}$, and $\lambda_{3}$ through the iterations as follows:

$$
\begin{gathered}
\lambda_{1}^{(n+1)}=\left[\lambda_{1}^{(n)}+\mu^{(n)}\left(\sum_{l=1}^{L} p_{l}\left(\lambda_{1}^{(n)}, \lambda_{2}^{(n)}, \lambda_{3}^{(n)}\right)-p_{\max }\right)\right]^{+}, \\
\lambda_{2}^{(n+1)}=\left[\lambda_{2}^{(n)}+\mu^{(n)}\left(\sum_{l=1}^{L} p_{l}\left(\lambda_{1}^{(n)}, \lambda_{2}^{(n)}, \lambda_{3}^{(n)}\right) h_{l}^{2}-\frac{I_{\max }}{1-\beta}\right)\right]^{+}, \\
\lambda_{3}^{(n+1)}=\left[\lambda_{3}^{(n)}+\mu^{(n)}\left(\sum_{l=1}^{L} p_{l}\left(\lambda_{1}^{(n)}, \lambda_{2}^{(n)}, \lambda_{3}^{(n)}\right) \zeta_{l}^{2}-\frac{\theta_{\max }}{1-\beta}\right)\right]^{+},
\end{gathered}
$$

where $n$ is the iteration index and $\mu^{(n)}$ is a sequence of scalar step size. Once $\lambda_{1}, \lambda_{2}$, and $\lambda_{3}$ are all obtained, we can get the optimal transfer power $p_{l}$ for $l=1,2, \ldots, L$ through (30).

The time complexity of the Lagrange multiplier algorithm depends on the estimation accuracy $\delta \in(0,1)$. By supposing that the stopping conditions of the iteration in (31) are $\mid \lambda_{1}^{(n+1)}$ $\lambda_{1}^{(n)}|\leq \delta,| \lambda_{2}^{(n+1)}-\lambda_{2}^{(n)} \mid \leq \delta$, and $\left|\lambda_{3}^{(n+1)}-\lambda_{3}^{(n)}\right| \leq \delta$, the iteration complexity is $O\left(1 / \delta^{3}\right)$, and the time complexity of each iteration is $O\left(L \log _{2} L\right)$. Hence, the aggregate time complexity of the Lagrange multiplier algorithm is given by

$$
F_{\mathrm{LM}}=O\left(\frac{L \log _{2} L}{\delta^{3}}\right) .
$$

The ADO method is used to obtain the optimal joint allocation of multislot spectrum sensing and transfer power by optimizing the two suboptimization problems (22) and (27) alternately and iteratively. The joint allocation algorithm based on ADO is described in Figure 7. Noting that the goal of optimizing (22) and (27) is to achieve the locally maximal value of the objective function, the value of the objective function is nondecreasing in each iteration as follows:

$$
C\left(\mathbf{k}^{(n-1)}, \mathbf{p}^{(n-1)}\right) \leq C\left(\mathbf{k}^{(n-1)}, \mathbf{p}^{(n)}\right) \leq C\left(\mathbf{k}^{(n)}, \mathbf{p}^{(n)}\right)
$$

where $n$ is the iteration index. Equation (31) indicates that if $C(\mathbf{k}, \mathbf{p})$ is convergent, both of $\mathbf{k}$ and $\mathbf{p}$ must be convergent. That is, if the stopping conditions of the iteration in ADO are $\left\|\mathbf{k}^{(n)}-\mathbf{k}^{(n-1)}\right\| \leq \delta$ and $\left\|\mathbf{p}^{(n)}-\mathbf{p}^{(n-1)}\right\| \leq \delta$, the optimal $C\left(\mathbf{k}^{(n)}, \mathbf{p}^{(n)}\right)$ can be obtained.

As the stopping conditions of the joint allocation algorithm are $\left\|\mathbf{p}^{(n)}-\mathbf{p}^{(n-1)}\right\| \leq \delta$ and $\left\|\mathbf{k}^{(n)}-\mathbf{k}^{(n-1)}\right\| \leq \delta$, the iteration complexity of the algorithm is $O\left(1 / \delta^{2}\right)$. In each iteration, the greedy algorithm with the complexity of $F_{\mathrm{GE}}$ and the Lagrange multiplier algorithm with the complexity of $F_{\mathrm{LM}}$ are both implemented once. Hence, the aggregate 


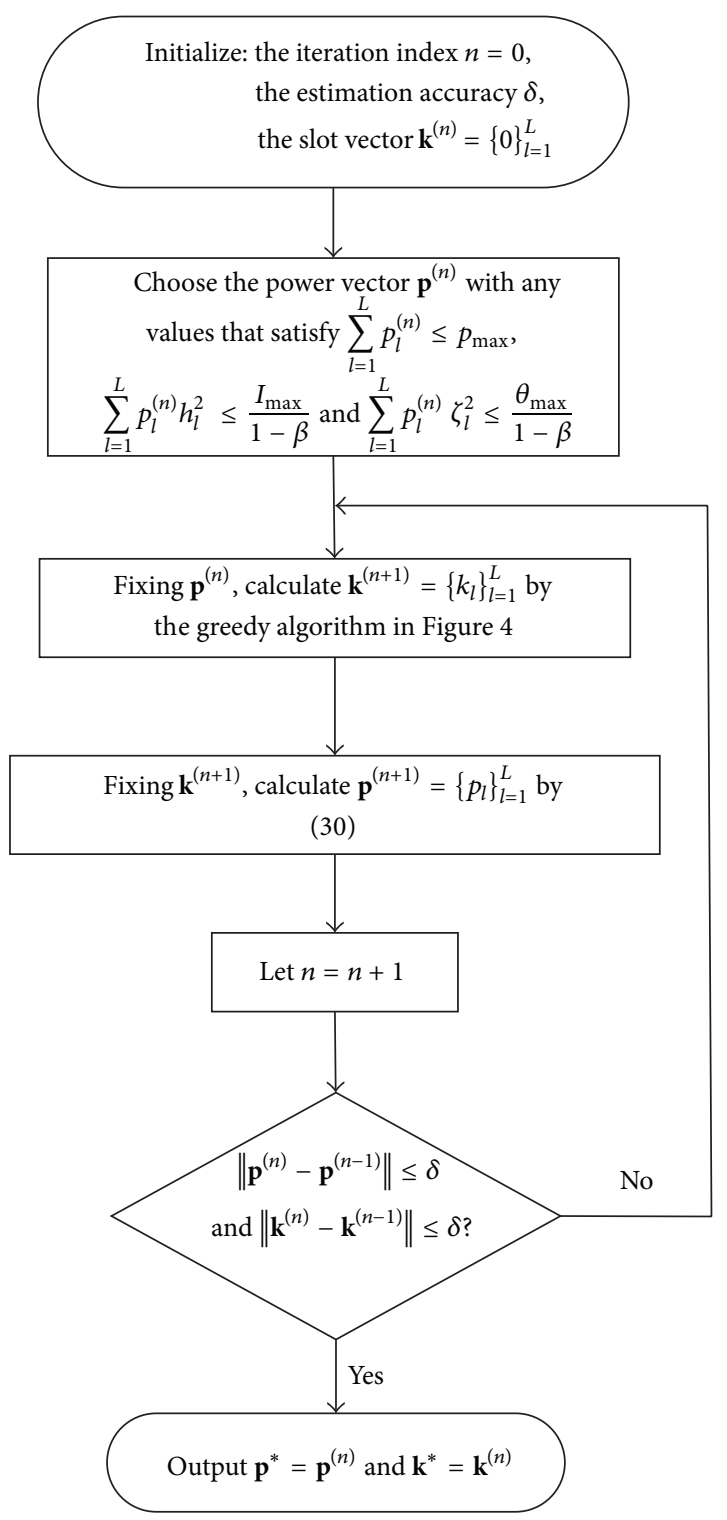

FIgURE 7: Joint allocation algorithm based on ADO.

time complexity of the joint allocation algorithm is given as follows:

$$
\begin{aligned}
& F_{\mathrm{JA}} \\
& =O\left(\frac{1}{\delta^{2}}\left(F_{\mathrm{GE}}+F_{\mathrm{LM}}\right)\right) \\
& =O\left(\frac{1}{\delta^{2}} \sum_{K=\sum_{l=1}^{L} q_{l}}^{\lceil T / \xi\rceil}\left(\left(K-\sum_{l=1}^{L} q_{l}\right) \log _{2} L+L\right)+\frac{L \log _{2} L}{\delta^{5}}\right) .
\end{aligned}
$$

However, since, in the conventional exhaustive searching method, we need to search through the vectors $\mathbf{k}$ and $\mathbf{p}$ including $2 L$ parameters, the time complexity of the exhaustive searching method is $O\left(1 / \delta^{2 L}\right)$ that greatly exceeds the time complexity of the proposed scheme if $L \geq 3$.

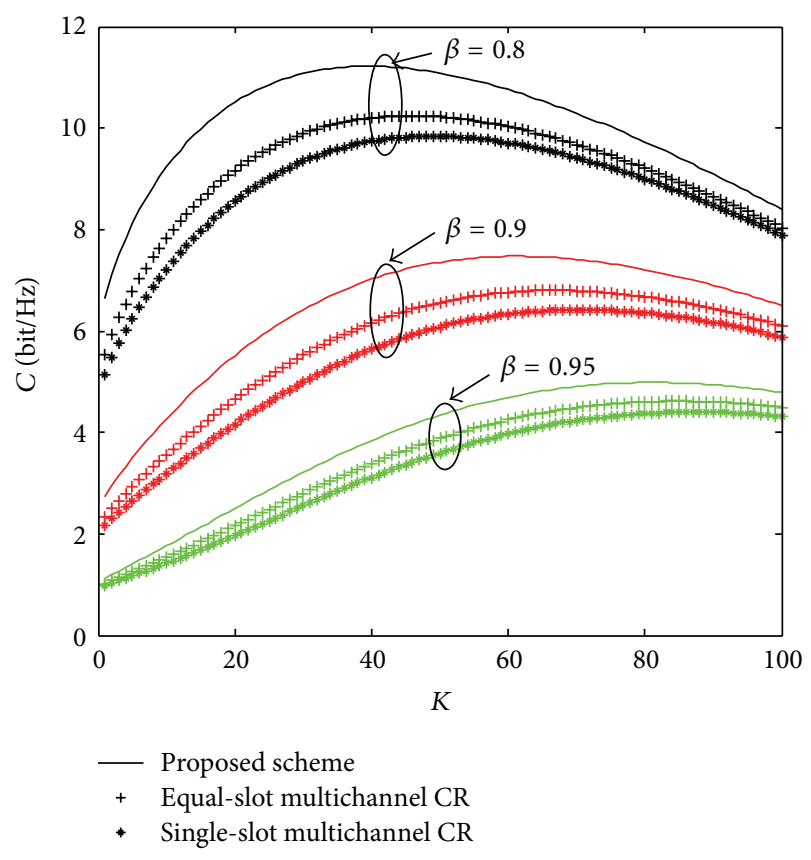

FigURE 8: Throughput versus lower bound of detection probability.

\section{Simulations}

In the simulation, the frame period $T=1 \mathrm{~s}$, the length of each time slot $\xi=5 \mathrm{~ms}$, the noise power $\sigma_{l}^{2}=0.01 \mathrm{~mW}$, the number of subchannels $L=16$, the subchannel gains $g_{l}$ for $l=1,2, \ldots, L$ obey the Rayleigh distribution with the mean of $-10 \mathrm{~dB}$, the sampling frequency $f_{s}=1 \mathrm{KHz}$, the maximal interference power and rate are $I_{\max }=10 \mathrm{~mW}$ and $r_{I}^{\max }=$ $1 \mathrm{bps} / \mathrm{Hz}$, respectively, the sensing SNR $\gamma_{l}$ for $l=1,2, \ldots, L$ is $[-10,-8,-6,-4,-2,-3,-5,-7,-9,-6,-3,-1,-2,-4,-6$, -8] $\mathrm{dB}$, and the appearance probabilities of the states $H_{0}$ and $H_{1} P_{H_{0}}=P_{H_{1}}=0.5$.

Figure 8 shows the SU's aggregate throughput $C$ versus the lower bound of detection probability $\beta$, with different number of sensing time slots $K$. From this figure, it is seen that there exists an optimal $K$ (the aggregate sensing time is $K \xi$ ) that maximizes $C$, and the convex-shaped $C$ is also consistent with Proposition 1 , which verifies the correctness of our allocation scheme. In the traditional equalslot multichannel CR of [10], the sensing time allocated to each subchannel is $K \xi / L$. With the same aggregate sensing time, the throughput of the proposed scheme is always larger than those of the equal-slot multichannel CR and the singleslot multichannel CR because, in our scheme, we allocate different sensing time to each subchannel according to their different gains. We also see that $C$ decreases greatly with the increase of $\beta$, because the false alarm probability increases with the increase of $\beta$, which reduces the SU's spectrum access. Figure 9 shows $C$ versus the maximal transfer power $p_{\max }$, with different $K$. It is seen that the proposed scheme outperforms the other two schemes, with various $p_{\max }$.

Figure 10 indicates the uniform-time throughput $C / T$ versus the frame period $T$, with different $K$. It is seen that 


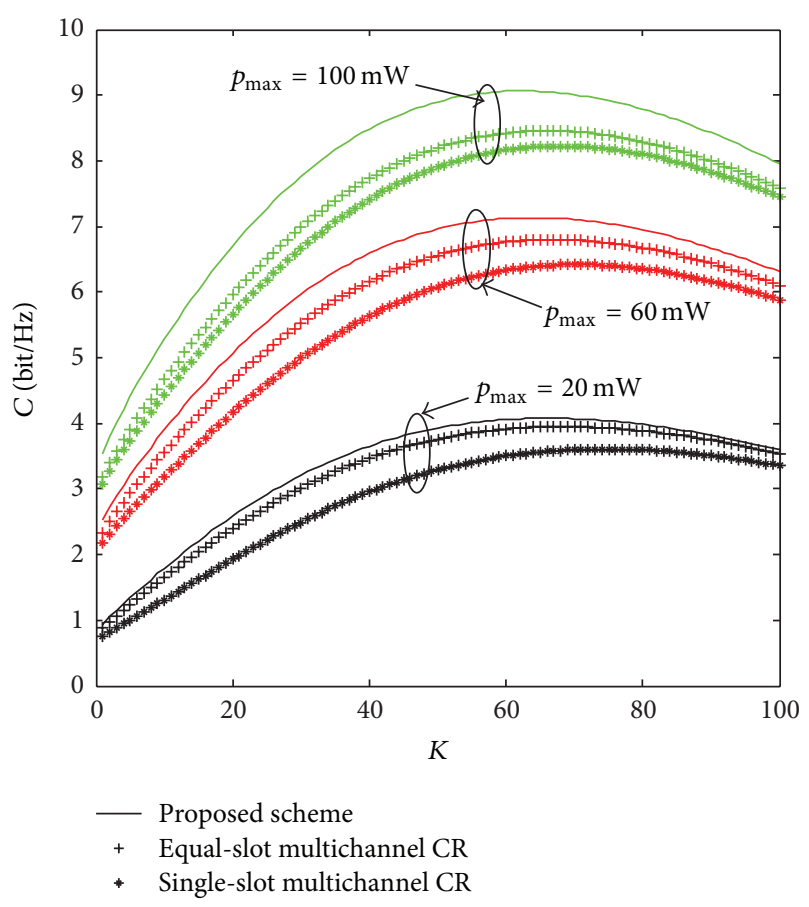

FIGURE 9: Throughput versus maximal transfer power.

with the increase of $T$, the number of the available sensing time slots increases, and longer sensing time can improve the SU's sensing performance. With the improved sensing performance, the decrease of the false alarm probability may increase the SU's spectrum access, and thus the SU's throughput is improved. Figure 11 compares the achieved throughput of the proposed scheme and the single-channel CR. It is seen that the proposed scheme that uses multiple subchannels to transfer can get much more throughput than the single-channel CR does.

Figure 12 shows the transfer power and false alarm probability of each subchannel by the proposed scheme. It is seen that in our allocation scheme, with the given detection probability, the larger power is allocated to the subchannels with lower false alarm probabilities, because the SU has more opportunities to access these subchannels, and larger power can help the SU make full use of the better subchannels in order to achieve perfect throughput. However, no power is allocated to the subchannels with much higher false alarm probabilities (i.e., subchannels 1 and 9), because the SU often cannot access these subchannels, and the transfer power allocation in these worse subchannels will not achieve benefits.

\section{Conclusions}

In this paper, we propose a multislot spectrum sensing and transfer scheme of multichannel CR. The frame is divided into sensing and transfer stages, and the sensing stage is further divided into many small time slots that are allocated to each subchannel for energy detection. An optimization problem of jointly allocating sensing time slots and transfer

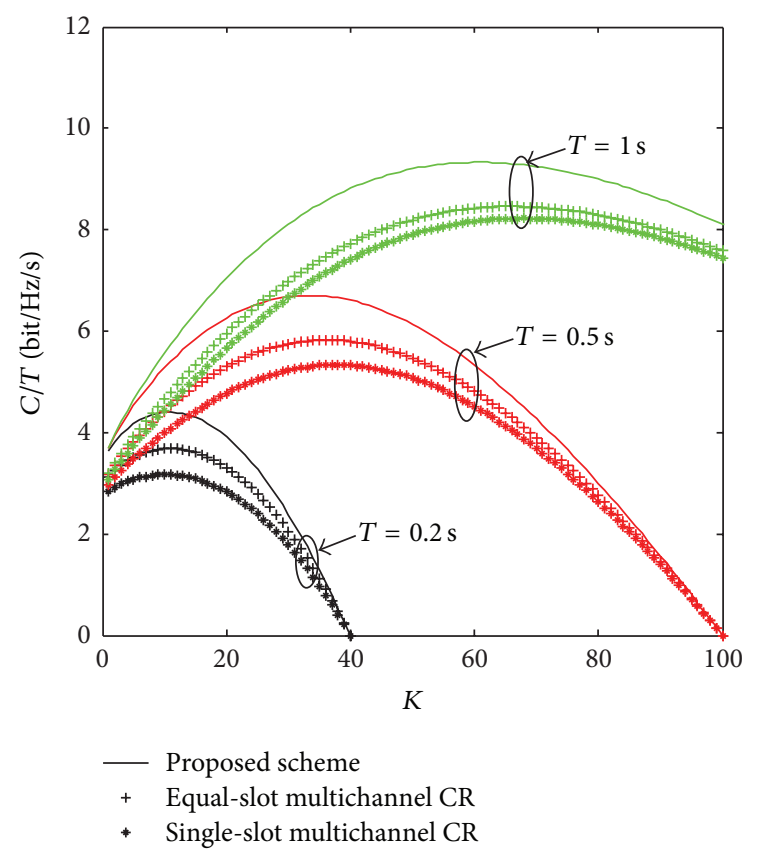

FIgURE 10: Throughput versus frame period.

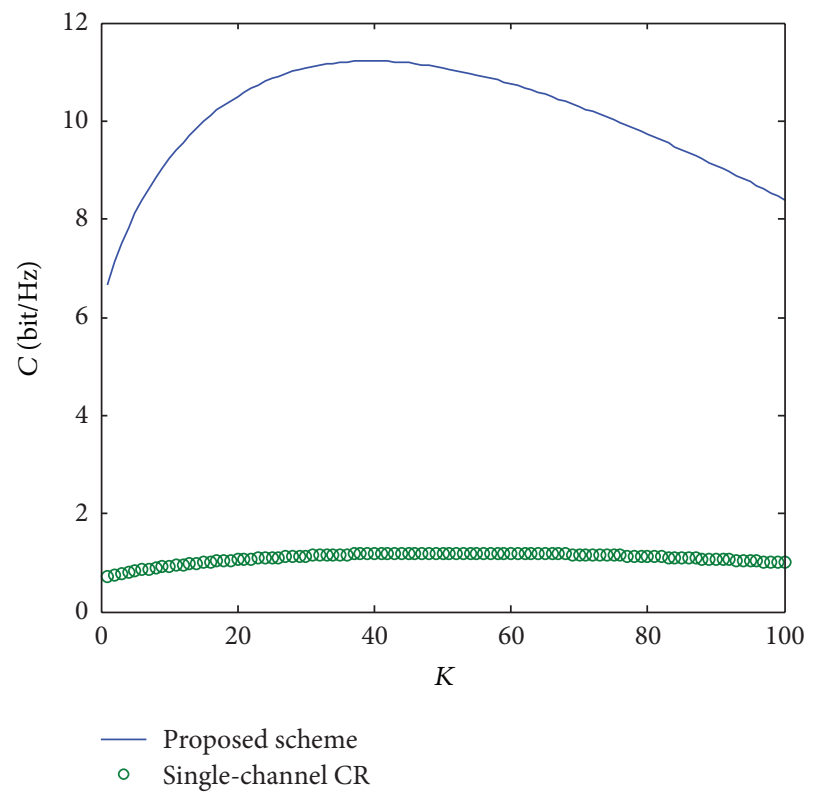

FIGURE 11: Throughput comparison of proposed scheme and singlechannel CR.

power to each subchannel is formulated to maximize the SU's aggregate throughput, subject to the constraints of the false alarm and detection probabilities of each subchannel and the aggregate interference power and rate of the SU. A joint allocation algorithm based on alternating directing optimization is proposed to solve the optimization problem. With numerical results, it is shown that there exists an optimal set of sensing time slots that maximizes the throughput 

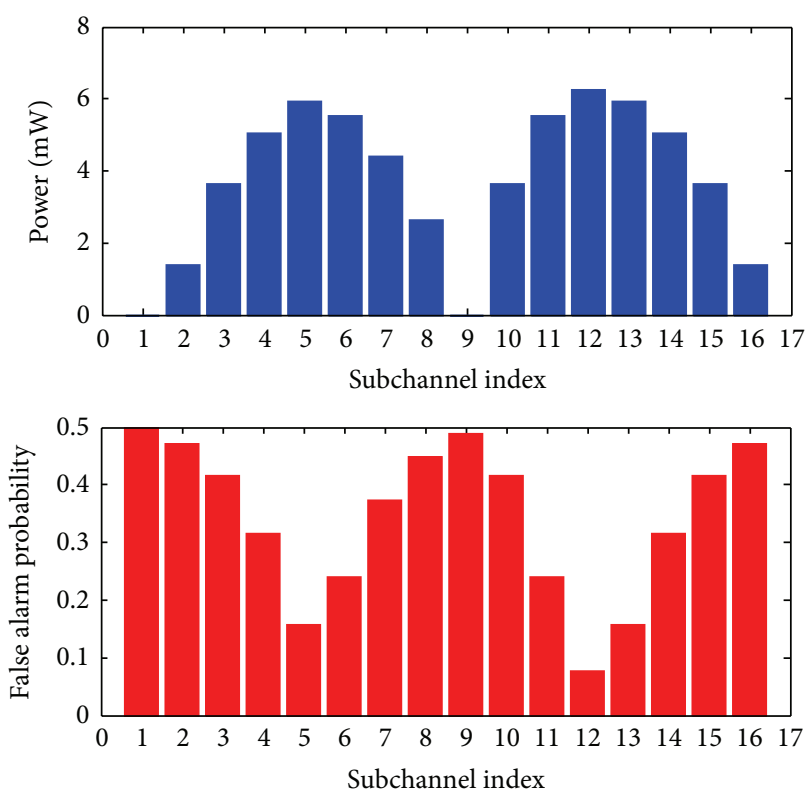

FIgURE 12: Power and false alarm probability of each subchannel.

and jointly optimizing sensing time slots and transfer power significantly improves the SU's aggregate throughput.

\section{Conflict of Interests}

The authors declare that there is no conflict of interests regarding the publication of this paper.

\section{Acknowledgments}

This work was supported by the National Natural Science Foundations of China under Grant nos. 61201143 and 61301105, the Natural Science Foundation of Jiangsu Province under Grant no. BK20140828, the Innovation Foundation of CAST (ITS) under Grant no. F-W-YY-2013-016, the Fundamental Research Funds for the Central Universities under Grant no. HIT.IBRSEM.201309, and the Scientific Research Foundation for Introduced Talent of Nanjing University of Aeronautics and Astronautics under Grant no. 56YAH13029.

\section{References}

[1] G. Baldini, O. Holland, V. Stavroulaki et al., "The evolution of cognitive radio technology in Europe: regulatory and standardization aspects," Telecommunications Policy, vol. 37, no. 2-3, pp. 96-107, 2013.

[2] D. Tandur, J. Duplicy, K. Arshad et al., "Cognitive radio systems evaluation: measurement, modeling, and emulation approach," IEEE Vehicular Technology Magazine, vol. 7, no. 2, pp. 77-84, 2012.

[3] H. Kremo and O. Altintas, "On detecting spectrum opportunities for cognitive vehicular networks in the tv white space," Journal of Signal Processing Systems, vol. 73, no. 3, pp. 243-254, 2013.
[4] H. Sun, A. Nallanathan, C. Wang, and Y. Chen, "Wideband spectrum sensing for cognitive radio networks: a survey," IEEE Wireless Communications, vol. 20, no. 2, pp. 74-81, 2013.

[5] X. Liu, J. Min, and G. Xuemai, "Joint optimal sensing threshold and subcarrier power allocation in wideband cognitive radio for minimising interference to primary user," China Communications, vol. 10, no. 11, pp. 70-80, 2013.

[6] A. Mariani, A. Giorgetti, and M. Chiani, "Effects of noise power estimation on energy detection for cognitive radio applications," IEEE Transactions on Communications, vol. 59, no. 12, pp. 34103420, 2011.

[7] X. Liu, M. Jia, X. Gu, and X. Tan, "Optimal periodic cooperative spectrum sensing based on weight fusion in cognitive radio networks," Sensors, vol. 13, no. 4, pp. 5251-5272, 2013.

[8] S. Gong, P. Wang, and J. Huang, "Robust performance of spectrum sensing in cognitive radio networks," IEEE Transactions on Wireless Communications, vol. 12, no. 5, pp. 2217-2227, 2013.

[9] R. F. Fan and H. Jiang, "Optimal multi-channel cooperative sensing in cognitive radio networks," IEEE Transactions on Wireless Communications, vol. 9, no. 3, pp. 1128-1138, 2010.

[10] Z. Quan, S. Cui, A. H. Sayed, and H. V. Poor, "Optimal multiband joint detection for spectrum sensing in cognitive radio networks," IEEE Transactions on Signal Processing, vol. 57, no. 3, pp. 1128-1140, 2009.

[11] X. Huang and B. Beferull-Lozano, "Joint optimization of detection and power allocation for OFDM-based cognitive radios," in Proceedings of the IEEE Global Telecommunications Conference, pp. 1-5, Miami, Fla, USA, December 2010.

[12] Y. Liang, Y. Zeng, E. C. Y. Peh, and A. T. Hoang, "Sensingthroughput tradeoff for cognitive radio networks," IEEE Transactions on Wireless Communications, vol. 7, no. 4, pp. 1326-1337, 2008.

[13] E. C. Y. Peh, Y. Liang, Y. L. Guan, and Y. Zeng, "Optimization of cooperative sensing in cognitive radio networks: a sensingthroughput tradeoff view," IEEE Transactions on Vehicular Technology, vol. 58, no. 9, pp. 5294-5299, 2009.

[14] W. Guibene and D. Slock, "Cooperative spectrum sensing and localization in cognitive radio systems using compressed sensing," Journal of Sensors, vol. 2013, Article ID 606413, 9 pages, 2013.

[15] M. Ferri, D. Pinna, E. Dallago, and P. Malcovati, "Photovoltaic energy harvester with power management system," Journal of Sensors, vol. 2010, Article ID 349389, 6 pages, 2010.

[16] N. T. Mohsen and U. Murat, "Sensing-throughput tradeoff in cooperative spectrum sensing," in Proceedings of the IEEE Global Communications Conference, pp. 1-6, Anaheim, Calif, USA, May 2012. 

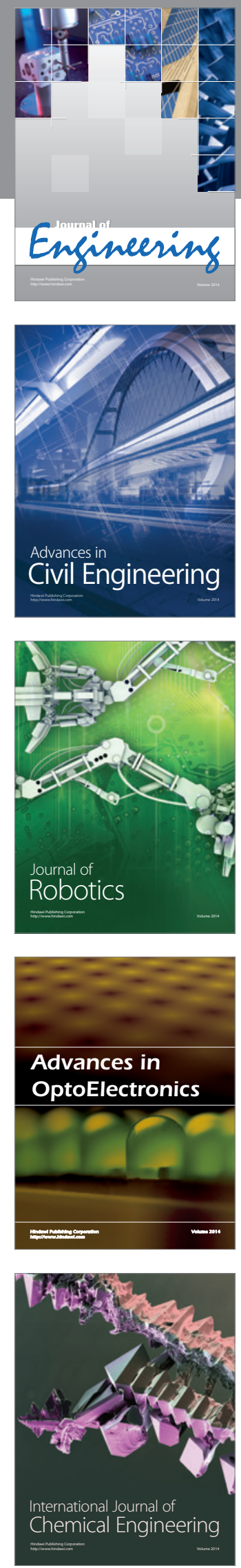

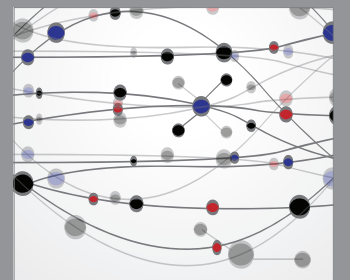

The Scientific World Journal
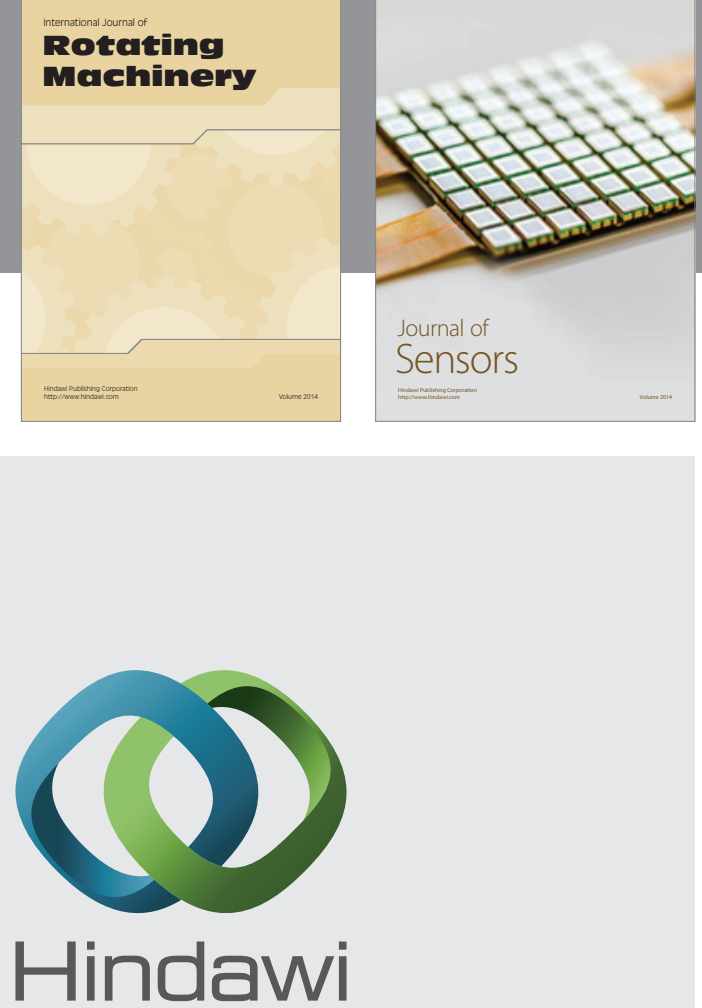

Submit your manuscripts at http://www.hindawi.com
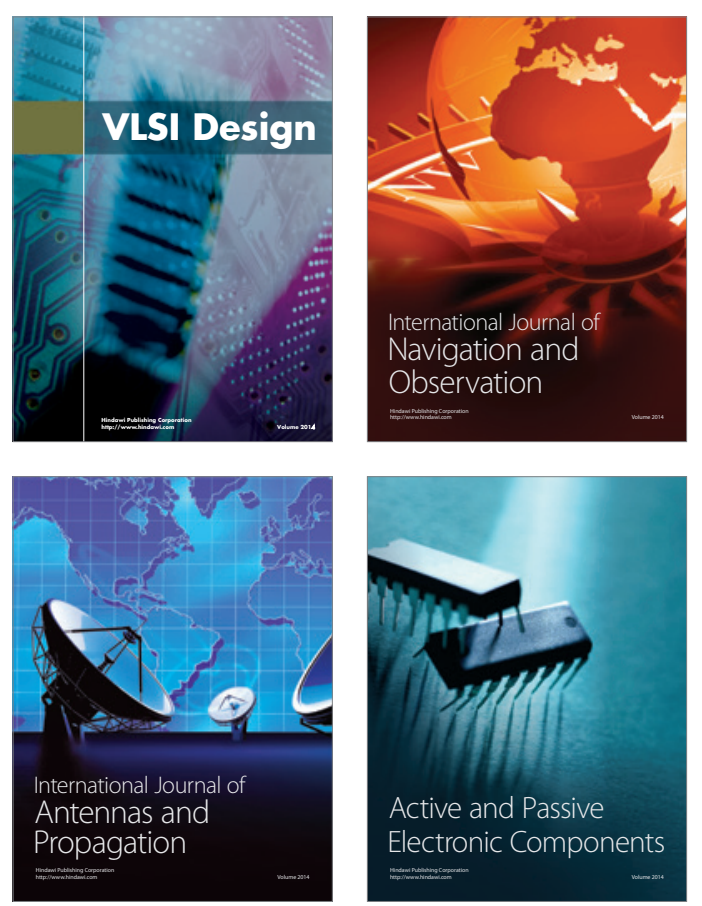
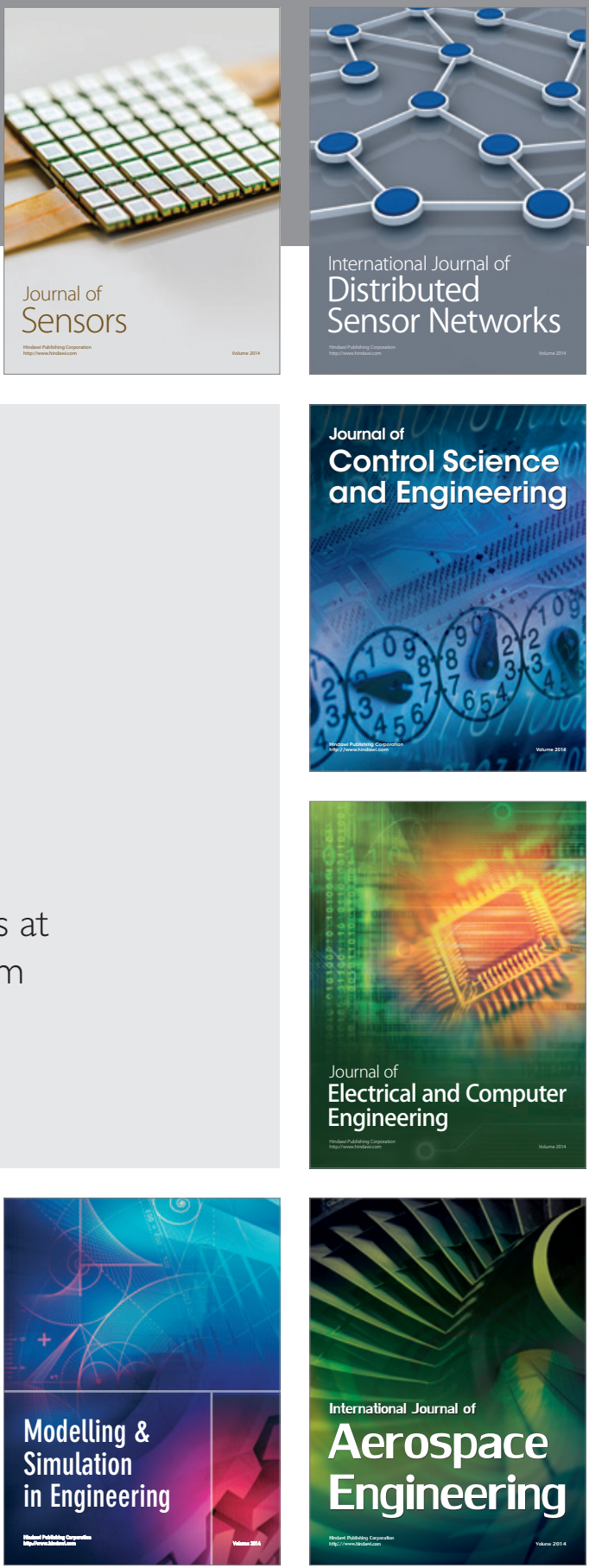

Journal of

Control Science

and Engineering
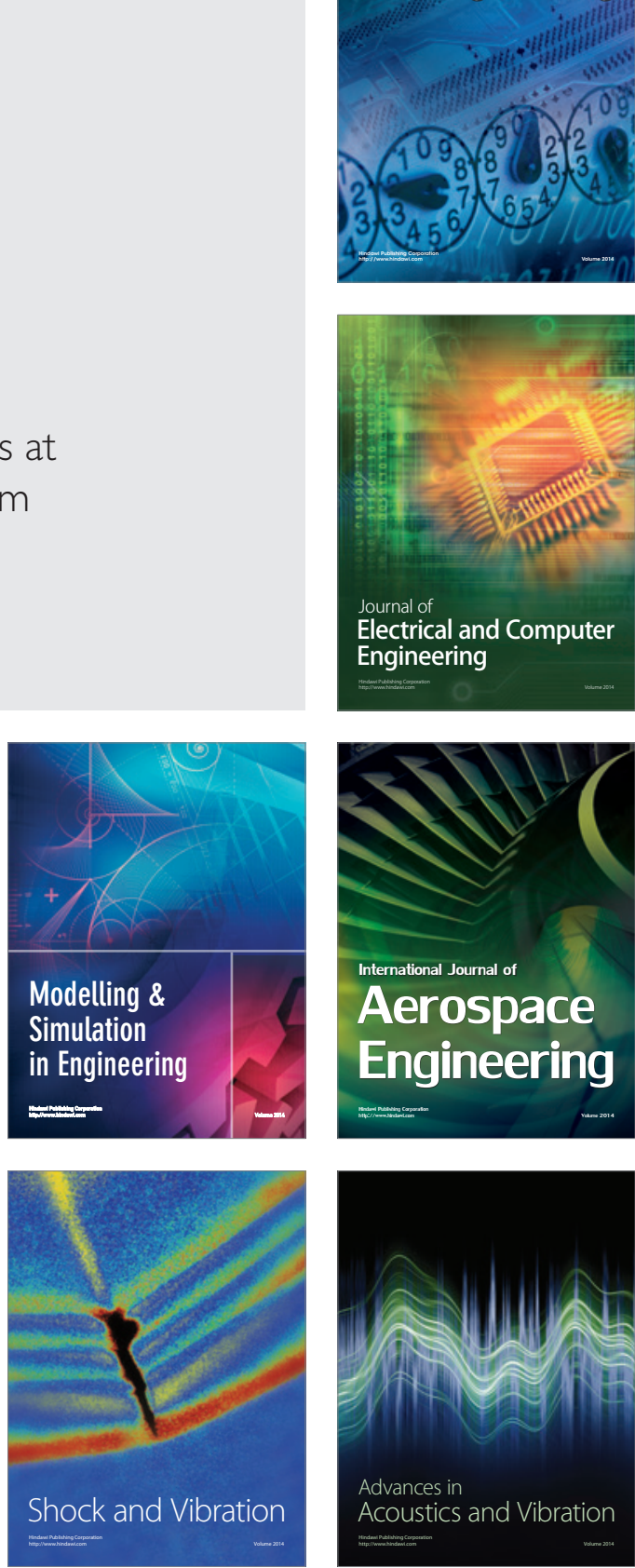